\title{
Impact of Subtle Gaze Direction on Short-Term Spatial Information Recall
}

\author{
Reynold Bailey* \\ Rochester Institute of Technology
Ann McNamara ${ }^{\dagger}$
Texas A\&M \\ Cindy Grimm ${ }^{\S}$ \\ Aaron Costello $\ddagger$ \\ Rochester Institute o Technology \\ Washington University in St. Louis
}

\begin{abstract}
The ability to direct a viewer's gaze about a scene has important applications in computer graphics and data visualization. The Subtle Gaze Direction (SGD) technique developed by Bailey et al. [2009] provides the ability to guide a viewer's gaze to specific regions of a display. The technique, which combines real-time eye-tracking with subtle image-space modulation, has minimal impact on viewing experience as it does not change the overall appearance of the scene being viewed. Subtlety is achieved by presenting the modulations only to the low-acuity peripheral regions of the field of view so the viewer is never allowed to scrutinize the modulations. The technique has been shown to be quite fast and accurate: viewers typically attend to target regions within 0.5 seconds of the onset of the modulation and the resulting fixations are typically within a single perceptual span of the target. While this shows that the technique is successful at directing gaze, it does not necessarily mean that the viewer fully processed the visual details of those regions or remembered them. To gain a better understanding of the level of visual processing involved, we conducted a study to determine the impact of SGD on short-term spatial information recall.
\end{abstract}

CR Categories: I.3.3 [Computer Graphics]: Picture/Image Generation-Display algorithms;

Keywords: Gaze direction, image-space, short-term memory

Links: DL 四PDF

\section{Approach}

Participants viewed a randomized sequence of 25 images. Following each image, they were presented with a blank screen and asked to recall the location of specific objects or regions. They were instructed to use the mouse to draw the smallest rectangles that bounded each target object or region. Their input was analyzed to determine how accurate their short-term spatial recollection was in terms of number of targets, location, and shape. 30 participants (4 females, 26 males), between the ages of 18 and 35 volunteered to participate in this study. Participants were randomly assigned to one of two groups:

\footnotetext{
*e-mail: rjb@cs.rit.edu

†e-mail: ann@viz.tamu.edu

¥e-mail: atc2436@rit.edu

§e-mail: cmg@cse.wustl.edu
}

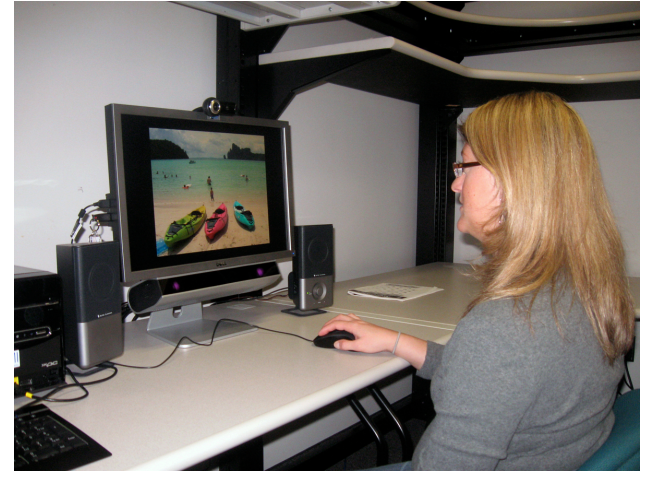

Figure 1: Photograph of experiment setup. The eye-tracking hardware is fixed to the bottom of the screen.

- Static group: 10 participants viewed the images without the use of gaze direction. This group served as the control group for the experiment.

- Gaze-directed group: 20 participants viewed the images with gaze direction turned on. For each image presented to the participants in this group, gaze direction was randomly selected to either direct the viewer's gaze away from the correct targets or direct the viewer's gaze to the correct target regions of the image. Counterbalancing was used to ensure that every image appeared equally often in both gaze-directed conditions.

\section{Results}

Our results show that the influence of SGD significantly improved accuracy of target count and spatial location recall. In particular, we observed the following effects:

- SGD to correct targets results in a significantly lower counting error compared to static viewing

- SGD to correct targets results in a significantly lower location error compared to static viewing

- SGD to incorrect targets results in a significantly higher location error compared to static viewing

- SGD has no significant impact on shape error

These results suggest that SGD plays a key role in aiding the prioritization of spatial attention thereby enabling the visual system to focus on key aspects of a scene while disregarding less salient information.

\section{References}

Bailey, R., McNamara, A., Sudarsanam, N., AND Grimm, C. 2009. Subtle gaze direction. ACM Trans. Graph. 28 (September), 100:1-100:14. 